\title{
\begin{tabular}{l|l} 
pcori)? & PATIENT-CENTERED OUTCOMES RESEARCH INSTITUTE \\
RESEARCH SUMMARY
\end{tabular}
}

\section{Using a Program to Lower Stress for Caregivers of Patients with Cancer Who Have Received Stem Cell Transplants}

Principal investigator

Mark L. Laudenslager, BA, PhD
Organization

University of Colorado Denver

\section{What was the research about?}

After having a stem cell transplant to treat cancer, patients often need help from a full-time caregiver at home. Support from a caregiver is important to a patient's recovery. But being a caregiver can be stressful. Stress management programs for caregivers may improve their well-being. Having a caregiver who is less stressed may also improve patients' quality of life.

The research team created a stress management program for caregivers of patients who received a stem cell transplant. The team wanted to see if the program helped caregivers and the patients they cared for. Caregivers in the program talked to a social worker about coping with stress and getting help from others when they felt stressed. The team compared people in the program with people getting usual support. Usual support included services such as support groups that hospitals offer as well as educational materials.

\section{What were the results?}

Caregivers in the program reported feeling less distressed and depressed compared with caregivers with usual support. There were no differences between caregivers in the two groups in other signs of well-being.

There were no differences in quality of life between patients with caregivers in the program and patients with caregivers getting usual support.

\section{Who was in the study?}

The study included 155 patients with cancer who had stem cell transplants and their caregivers. Patients received care from a hospital or a health center in Denver, Colorado. Of the caregivers, 83 percent were white, 6 percent were another race, and 4 percent were multiracial. Also, 7 percent were Hispanic. The average caregiver age was 54 , and 80 percent were women.

\section{What did the research team do?}

This study put caregivers into one of two groups by chance. The first group was in the stress management program. The second group had usual support.

Caregivers in the program had 8 to 10 sessions with a social worker in the three months after the patients' transplants. About 15 percent of sessions were by telephone or video chat. The rest were in-person sessions.

The research team followed up with patients and caregivers at six weeks, three months, and six months after the patients' transplants. Caregivers answered questions about depression, anxiety, stress, and burden. They also gave blood and hair samples to test for signs of stress. Patients took surveys about quality of life.

A community advisory board gave input during the study. The board included six former patients and their caregivers and two members of a community support group. 


\section{What were the limits of the study?}

This study took place in the Denver area. Most caregivers were white women in their 50s. Results may have been different for caregivers from other backgrounds or who live in other places. Results also may have been different for patients with other cancers or who didn't have stem cell transplants.

Future research could look at ways to further lower caregiver stress and include patients and caregivers with different backgrounds.

\section{How can people use the results?}

Hospitals can look at these results when deciding on ways to help lower caregivers' stress.

To learn more about this project, visit www.pcori.org/Laudenslager146. 